\title{
Abordaje de los trabajadores con trastornos físicos y psíquicos en salud laboral: un modelo
}

Approach of workers with physical and mentaldisorders in occupational health: a model

\section{Fernando Mansilla Izquierdo}

Madrid-Salud. Madrid. España.

\section{Juan Carlos García Micó}

Madrid-Salud. Madrid. España.

\section{Casimiro Gamero Merino}

Madrid-Salud. Madrid. España.

\section{Aurora Congosto Gonzalo}

Madrid-Salud. Madrid. España.

\section{Cristina Calderón Congosto}

Universidad Rey Juan Carlos. Madrid. España.

\section{Rodrigo Gamero Navarro}

Hospital del Henares. Madrid. España.

\section{Recibido: 20-10-10}

Aceptado: 25-1 1-10

\section{Correspondencia:}

Fernando Mansilla Izquierdo

C/ Emilio Coll, 22, 1

28224- Pozuelo de Alarcón (Madrid).

Tfno: 917140458

Fax: 915883165

Correo electrónico: mansillaif@munimadrid.es

Resumen

En este artículo se propone un modelo de abordaje en salud laboral de los trabajadores con trastornos físicos o psíquicos, utilizando la entrevista motivacional y el apoyo social como ejes del modelo. La atención se realiza en tres fases: contención, valoración y orientación. Este modelo puede favorecer la disminución de los problemas o dificultades en el contexto laboral, adaptando o cambiando su puesto de trabajo.

Med Segur Trab (Internet) 2010; 56 (221): 274-279

Palabras clave: Modelo. Entrevista Motivacional. Apoyo Social. Salud laboral.

Abstract

This article proposes a model approach to occupational health of workers with physical or mental disorders, using motivational interviewing and social support model axis. Attention is done in three phases: containment, assessment and guidance. This model may help to decrease the problems or difficulties in the employment context, adapting or changing their jobs.

Med Segur Trab (Internet) 2010; 56 (221): 274-279

Key words: Model. Motivational Interviewing. Social Support. Occupational Health. 


\section{INTRODUCCIÓN}

En algunas ocasiones los trabajadores que acuden a Salud Laboral manifiestan las consecuencias psíquicas o físicas de estrés o crisis debidas a problemas médicos, psicopatológicos o derivados del contexto laboral, ya que la aparición de la enfermedad en la vida de una persona supone siempre una situación de crisis y un deterioro de su calidad de vida (Rodríguez-Marín, Pastor y López-Roig, 1993) y, por tanto, del contexto laboral. Y el tipo de respuesta más habitual lo constituye la depresión y la ansiedad (Rodríguez-Marín, Pastor y López-Roig, 1993).

Los trabajadores reaccionan de manera diferente frente a situaciones de estrés y crisis sobre la base de sus propias habilidades, nivel de maduración y personalidad.

Se ha definido la crisis como una amenaza a la homeostasis, y produce un desequilibrio que se traduce en confusión y desorganización (Caplan, 1961). Se desencadena cuando un acontecimiento vital estresante desafía la capacidad del individuo para hacerle frente de manera efectiva. Y se puede generar porque la homeostasis psicológica se ha visto alterada o porque los mecanismos de supervivencia no han logrado restablecerla (Everly y Mitchell, 1999). Es decir, una crisis puede referirse a cualquier situación en la que el individuo percibe una pérdida repentina de sus habilidades de afrontamiento y de su capacidad de resolución de problemas.

\section{ESTRATEGIA}

La intervención se conjugará sobre dos ejes: la entrevista motivacional y el apoyo social.

La entrevista motivacional es un tipo de entrevista centrada en el cliente, de estilo directivo para la preparación al cambio, con una evolución sobre el enfoque de Counseling (asesoramiento) de Carl Rogers (1997), que busca ayudar a explorar y resolver contradicciones sobre conductas o hábitos insanos e intenta aumentar la conciencia sobre los riesgos de salud y la capacidad para hacer algo al respecto: Es decir, es una técnica terapéutica que tiene por objetivo implicar a la persona en un proceso de cambio de conducta, ayudándole a desarrollar sus propias habilidades para el cambio (Miller, 1983; Miller y Rollnick, 1991, 2002; Hettema, Steele y Miller, 2005; Rollnick, Miller y Butler, 2008; Lizarraga y Ayarra, 2001). Además hay que tener en cuenta que la motivación para el cambio es mayor si hay un delicado proceso de negociación entre el terapeuta y el trabajador, y es el trabajador el que valora los beneficios y los costos involucrados. Además, el conflicto no es útil, es más positiva una relación de colaboración entre el terapeuta y el trabajador (Treasure, 2004). Para ello, es conveniente realizar preguntas abiertas para facilitar la expresión de los sentimientos y los pensamientos, ejercer la escucha reflexiva, hacer resúmenes de lo que se va verbalizando y destacar los aspectos positivos y de cambio (Miller, 1983; Miller y Rollnick, 1991, 1996; Rollnick, Miller y Butler, 2008). Y en la relación con el trabajador no se tiene que dogmatizar, generalizar, minimizar, moralizar, hacer monólogos, ser inquisitivo, etc.

La entrevista motivacional (Miller, 1983; Miller y Rollnick, 1991, 2002; Rollnick, Miller y Butler, 2008; Lizarraga y Ayarra, 2001) consta de una serie de elementos teóricos y prácticos que tratan de mejorar las habilidades de comunicación. Se basa en cinco principios: Expresar empatía, Elaborar la ambivalecia o discrepancias (para aclarar las dualidades que pueda haber en su demanda), Evitar la confrontación y las discusiones, Lidiar o esquivar las resistencias y Apoyar o reforzar la autoeficacia.

También se ha señalado que el apoyo social protege contra el desarrollo de problemas mentales y físicos cuando el individuo está expuesto a factores de estrés, como los acontecimientos vitales negativos (Schwarzer y Leppin, 1989; Helgeson y Cohen, 1996; Sánchez y Barrón, 2003). Y en general se supone que el apoyo social tiene un impacto 
favorable en el mantenimiento de la salud y para hacer frente a la enfermedad (Schwarzer y Leppin, 1991; Mansilla, 1993; Clingerman, 2004; Fernández Peña, 2005).

En la intervención se tendrá en cuenta el apoyo social que son los vínculos entre individuos que sirven para improvisar competencias adaptativas en el manejo de crisis y problemas inmediatos (Caplan, 1974).

Se han distinguido cuatro tipos de apoyos: emocionales, instrumentales, de información y de valoración (House, 1981). Y se ha señalado que el apoyo social se compone de cuatro factores: orientación directiva, ayuda no directiva, interacción social positiva y ayuda tangible (Barrera y Ainlay, 1983). También se ha dado un gran valor a la información cuando se recibe de una persona significativa (Gore, 1981).

Es necesario destacar la importancia de la percepción del apoyo social, porque la realidad para el individuo es fundamentalmente la realidad percibida. De modo que el apoyo percibido se puede identificar como: información de que el sujeto es querido, información de que el sujeto es estimado y valorado e información de que el sujeto posee capacidad para establecer redes de comunicación y de relación (Cobb, 1976).

Por otro lado, el efecto amortiguador del apoyo social actúa modificando las respuestas negativas frente al estrés, facilitando recursos y permitiendo una reevaluación del acontecimiento y una positiva adaptación (Gore, 1981; Cohen y Wills, 1985; Alloway y Bebbington, 1987; Barrón, 1996).

De manera que el apoyo social puede contribuir a favorecer que los trabajadores en crisis, estrés o con dificultades resuelvan problemas y recuperen el equilibrio emocional y laboral.

\section{FASES DE LA INTERVENCIÓN}

La intervención de apoyo es un proceso de comunicación interpersonal entre un profesional y un sujeto necesitado de ayuda por problemas, que tiene por objeto producir cambios para mejorar la salud.

Dentro de una planificación sistemática y estructurada que es la Atención en la Salud Laboral, el abordaje de cada trabajador es específico, flexible y contextualizado.

La intervención se realiza en varias sesiones, y es enfocada desde una perspectiva multidisciplinar y realizada en equipo. Esta intervención inmediata de apoyo tiene como objetivo restablecer una cierta estabilidad emocional, y pueden realizarla los diferentes miembros del equipo.

Este modelo individualizado de intervención que se centra en el aquí y ahora, suele incluir la psicoeducación de las implicaciones de su problema y síntomas en el contexto laboral, el asesoramiento en los pasos a seguir y la facilitación de los apoyos destinados a ofrecer las ayudas necesarias para compensar o fortalecer el nivel de funcionamiento psicolaboral.

La intervención debe estar orientada a un apoyo social y afectivo para superar el estrés o la crisis y restablecer el equilibrio emocional. Este apoyo se realiza mediante la creación de una relación en la que el paciente no se sienta juzgado, ni marginado, sino entendido y ayudado para poder confrontar, desde la acogida y el respeto, sus temores y necesidades.

Esta intervención se realiza de forma inmediata a los trabajadores que pasan por una situación emocional crítica, sea o no debido al trabajo, para que restablezcan su estabilidad emocional y facilitarle las condiciones del ambiente laboral.

Tiene tres fases: a. Contención, b. Valoración y c. Orientación. 


\section{a. Contención:}

En esta fase la relación con el paciente que se construya debe ser una relación empática, que trasmita confianza, para que el paciente se sienta cómodo y sienta que nos preocupamos por su problema. Es conveniente tener una actitud comprensiva y firme, pero sin crítica (no se juzga, ni se interroga, ni se aconseja, ni se opina). También hay que favorecer un ambiente de apoyo y aceptación incondicional, recogiendo los sentimientos contradictorios de la persona.

En la entrevista se ofrece un tiempo y espacio donde es posible reflexionar y ventilar los conflictos. Y donde puede generarse un clima de confianza, de apertura y de sincera comunicación, es decir, una relación mutuamente comprensiva donde pueda vencerse la resistencia (la resistencia es una forma de respuesta debido al miedo o la ansiedad, evitando un estado potencial de estrés o crisis y admitirse un cambio de actitud estable). También ofrece la primera ayuda emocional a corto plazo a las personas que tienen experiencias con consecuencias emocionales, mentales, físicas o problemas de comportamiento. Y se promueve el cambio psíquico, buscando aliviar el sufrimiento y favorecer el desarrollo de la persona y de sus vínculos (García Ramos y Gallego Rodríguez, 2003).

En el primer contacto tiene que procurarse una sintonía con los sentimientos y necesidades del trabajador. La tarea es escuchar cómo la persona visualiza su situación. Así mismo, se le invita a hablar sobre lo ocurrido, a escucharse y a darse cuenta de sus reacciones (sentimientos y actitudes) ante el acontecimiento, estableciendo lineamientos reflexivos. En esta fase se facilita a los trabajadores a hablar de lo que les sucede a nivel físico o psíquico, lo que puede ser importante para la reducción de la ansiedad. Es decir, el trabajador debe sentir que se le escucha, acepta, entiende y apoya; lo que a su vez conduce a la expresión de sentimientos y a la disminución en la intensidad de sus ansiedades.

\section{b. Valoración:}

Se trata de realizar la evaluación funcional de las capacidades y habilidades del trabajador en relación con las demandas del puesto de trabajo en su entorno concreto.

Para ello, se realiza la recopilación de información y se analizan las dimensiones del problema. La información que se recopila se enfoca sobre el presente (cómo se siente, qué piensa), sobre el pasado inmediato (qué sucedió, qué acontecimientos llevaron al estado de estrés o crisis) y sobre el futuro inmediato (qué puede hacer).

Se tienen que valorar las áreas fisiológica, cognitiva y motora, teniendo en cuenta la reacción y el impacto que le causa hablar del incidente crítico.

Esta fase implica generar alternativas y estrategias ante los problemas que plantee y una evaluación de necesidades del trabajador para determinar los servicios y recursos requeridos. Por tanto, se analizan los obstáculos para la ejecución de un plan en particular y se sondean las posibles soluciones.

\section{c. Orientación:}

En esta fase se fomenta la implicación y participación activa del trabajador y se planifica e identifica con claridad las soluciones al problema. Se proporciona la información sobre los pasos a seguir y los recursos y servicios. Y hay que tomar las decisiones prácticas, iniciando los pasos concretos, que conducen a ayudar al trabajador a ejecutar la acción concreta, con una actitud facilitadora o directiva, según las circunstancias.

\section{DISCUSIÓN Y CONCLUSIONES}

En este artículo se ha desarrollado un modelo de intervención para los problemas físicos y psíquicos en Salud Laboral. 
Esta intervención es la prestación de apoyo social para ayudar a los trabajadores a regresar a un nivel de adaptación de funcionamiento y para prevenir o mitigar los posibles efectos negativos del impacto del problema. Es decir, que se centra en el funcionamiento del trabajador para apoyar el desempeño de su rol laboral y, por tanto, pretende mejorar su calidad de vida.

El apoyo psicológico está indicado en todo tipo de pacientes, en todo tipo de enfermedades, y la duración no está limitada al tiempo de la sesión o al tiempo del profesional, sino que puede adaptarse a las necesidades del paciente (Ordoñez Fernández, 2008), de ahí que sea también beneficioso en la atención en salud laboral.

Aunque la entrevista motivacional originalmente se desarrolló para los bebedores, se ha utilizado con una amplia gama de comportamientos y de poblaciones, incluyendo problemas de adicciones, tabaquismo (Balcells Olivero, Torres Morales y Yahne, 2004), en el abandono del tratamiento antipsicótico (González Varea, 2006), como instrumento para promover la actividad física y la adhesión dietética en personas con diabetes (Leyva Moral, 2007) y en Atención Primaria (Ruiz Lázaro, 2009; Puschel y otros, 2006; Rivera Mercado, Villouta Cassinelli e Ilabaca Grez, 2008), entre otras, además puede ser utilizada en otros múltiples problemas en Salud Laboral, que junto con un enfoque de apoyo social se ha demostrado beneficioso para los trabajadores.

Así como a la entrevista motivacional le dio la columna vertebral el modelo transteórico de cambio (Prochaska y DiClemente, 1986; Prochaska y Norcross, 1994; Prochaska y Velicer, 1997) que propone varias etapas: Precontemplación, Contemplación, Preparación, Acción, Mantenimiento, el modelo que se propone es una intervención teniendo como ejes la entrevista motivacional y el apoyo social en varias fases: Contención, Valoración y Orientación.

Asimismo, durante la entrevista motivacional la muestra del apoyo social que amortigua los efectos del estrés en los individuos, como parte de la atención a los trabajadores con problemas físicos o psíquicos, favorece la disminución de los problemas o dificultades y se ponen las bases para la integración en el contexto laboral, adaptando o cambiando su puesto de trabajo.

\section{BIBLIOGRAFÍA}

Alloway, R. y Bebbington, P. (1987). The buffer theory of social support: a review of the literature. Psychological Medicine, 17, 1, 91-108

Balcells Olivero, M., Torres Morales, M. y Yahne, C.E. (2004). La terapia motivacional en el tratamiento del Tabaquismo. Adicciones, 16, 2 227-236.

Barrera, M. y Ainley, S.L. (1983). The structure of social support: A conceptual and empirical analysis. Journal of Community Psychology, 11, 133-144.

Barrón, A. (1996). Apoyo Social. Madrid: Siglo XXI de España Editores.

Caplan, G. (1961). A approach to Community Mental Health. New York:Grunne \& Stratton.

Caplan, G. (1974). Support Systems and Community Mental Health. New York: Behavioral Publications.

Clingerman, E. (2004). Physical Activity, Social Support and Health-Related Quality of Life Among Persons with HIV Disease. Journal of Community Health Nursing, 21, 3, 179-197.

Cobb, S. (1976). Social support as a moderator of Iife events. Psychosomatic Medicine, 38, 5, 300-314.

Cohen, S. y Wills, T. A. (1985). Stress, social support, and the buffering hypothesis. Psychological Bulletin, 98, 310-57.

Everly, Jr., G.S. y Mitchell, J.T. (1999). Critical incident stress management (CISM): A new era and standard of care in crisis intervention (2nd Ed.). Ellicott City, MD: Chevron.

Fernández Peña, R. (2005). Redes Sociales, apoyo social y salud. Revista de recerca i investigació. Periferia, 3 www.periferia.name.

García Ramos, J. y Gallego Rodríguez, J. M. (2003). Psicoterapias en Atención Primaria. Terapéutica en APS. FMC; 10, 7, 508-513. 
González Varea, J. (2006). Abandono del Tratamiento Antipsicótico: Entrevista Motivacional. Presentado en el 7. ${ }^{\circ}$ Congreso Virtual de Psiquiatría. Interpsiquis. www.psiquiatria.com.

Gore, S. (1981). Stress-Buffering functions of social support: An Appraisal and c1arification of research models. En B.S. Dohrenwend y B.P. Dohrenwend (Eds.). Stressfullife events and their contexts. New York: Prodist.

Helgeson, V. y Cohen, S. (1996). Social support and ajustment to cancer: reconciling descriptive, corretional, and intervention research. Health Psychology, 15, 135-148.

Hettema, J., Steele, J. y Miller, W.R. (2005). Motivational Interviewing. Annual Review of Clinical Psychology, 1, 91-111.

House, J.J. (1981). Work stress and social support. Reading MA: Addison-Wesley.

Jacobson, G., Strickler, M. y Morley, W. (1968). Generic and individual approaches to crisis intervention. American Journal of Public Health, 58, 338-343.

Leyva Moral, J.M. (2007). La entrevista motivacional como instrumento para promover la actividad física y la adhesión dietética en personas con diabetes: revisión bibliográfica. Nure Investigación, 29, 35-39.

Lizarraga, S. y Ayarra, M. (2001). Entrevista Motivacional. Anales Sts San Navarra, 24, 2, 43-53.

Mansilla, F. (1993). Red Social y Apoyo Social en Enfermos Mentales Sin Hogar. Revista de la Asociación Española de Neuropsiquiatría. 45, 46-51.

Miller, W.R. (1983). Motivational interviewing with problem drinkers. Behavioural Psychotherapy, 11, 147172. (Descripción original de este método).

Miller, W.R. y Rollnick, S. (1996). ¿Qué es la entrevista motivacional? RET, Revista de Toxicomanias, 6, 5-9.

Miller, W.R. y Rollnick, S. (2002). Motivational interviewing: Preparing people for change (2nd ed.). New York: The Guilford Press.

Ordóñez Fernández, M.P. (2008). Psicoterapia de Apoyo en Atención Primaria. Revista Clínica de Medicina de Familia, 2, 5, 13-19.

Prochaska, J.O. y DiClemente, C.C. (1986). Toward a comprehensive model for change. In Miller WR and Heather N (Eds). Treating addictive behaviors. New York, NY: Plenum Press.

Prochaska, J. O. y Norcross, J. (1994). Systems of Psychotherapy: A Transtheoretical Analysis. Pacific Grove, CA: Brooks/Cole Publishing.

Prochaska, J.O. y Velicer, W.F. (1997). The transtheoretical model of health behavior change. American Journal of Health Promotion, 12, 38-48.

Puschel, K., Thompson B., Coronado G., Rivera, S., Díaz, D., González, L., Valencia, G., Iñiguez; S., Montero, J. (2006). Tabaquismo en Atención Primaria: Perfil de fumadoras consultantes, creencias y actitudes de los equipos de salud y oportunidades de intervención. Revista Médica de Chile, 134, 726-734.

Rivera Mercado, S., Villouta Cassinelli, M.F. e Ilabaca Grez, A. (2008). Entrevista motivacional: ¿̨uál es su efectividad en problemas prevalentes de la atención primaria? Atención Primaria: Publicación oficial de la Sociedad Española de Familia y Comunitaria, 40, 5, 257-261

Rodríguez-Marín, J., Pastor, M.A. y Lopez-Roig, S. (1993). Afrontamiento, apoyo social, calidad de vida y enfermedad. Psicothema, 5, 1, 349-372.

Rogers, C. (1997). Psicoterapia centrada en el cliente. Barcelona: Ediciones Paidós Ibérica

Rollnick, S., Miller, W.R. y Butler, C. (2008). Motivational Interviewing in Health Care. Helping. Patients. Change. Behavior. New York: The Guilford Press.

Ruiz Lázaro, P.J. (2009). Dos herramientas para entrevistar adolescentes: la entrevista motivacional y el consejo sociosanitario participativo. Revista de Pediatría en Atención Primaria, 11, 41, 21-27.

Sánchez, E. y Barrón, A. (2003). Social psychology of mental health: the social structure and personality prespective. The Spanish Journal of Psychology, 6, 3-11.

Schwarzer, R. y Leppin, A. (1989). Social Support and Health: A meta-analysis. Psychology and Health, 3, 1-15.

Schwarzer, R. y Leppin, A. (1991). Social Support and Health: A Theoretical and Empirical Overview. Journal of Social and Personal Relationships, 8, 99-127.

Treasure, J. (2004). Motivational Interviewing. Advances in Psychiatric Treatment, 10, 331-337. 